\title{
AGROPECUÁRIA NA REGIÃO NORTE DO BRASIL: TRANSBORDAMENTOS TECNOLÓGICOS ENTRE MUNICÍPIOS
}

\author{
Edi Flores Reyna ${ }^{1}$ \\ Rubicleis Gomes da Silva ${ }^{2}$ \\ Viviani Silva Lírio ${ }^{3}$
}

Resumo: Este trabalho tem como objetivo verificar a existência do efeito transbordamento de tecnologia do setor agropecuário entre os municípios da região Norte, bem como analisar os determinantes do valor de produção do setor em 2006. Para esse fim, foram utilizados métodos derivados da econometria espacial. Os resultados obtidos mostraram que no setor agropecuário da região Norte a variável mão-de-obra possui maior impacto sobre o valor de produção e, que não existe transbordamento de tecnologia entre os municípios. Este pode ser explicado pela baixa densidade rodoviária na região e pela distância existente entre os estabelecimentos que não permitem a disseminação e compartilhamento de tecnologia. Contudo, verificou-se a existência de dependência espacial no termo de erro na agropecuária nortista.

Palavras-chave: Agropecuária. Função de produção. Externalidade. Transbordamento.

\section{AGRICULTURE IN THE NORTHERN REGION OF BRAZIL: SPILLOVERS OF TECHNOLOGY AMONG MUNICIPALITIES}

Abstract: This paper, about the northern agricultural sector, aims to verify the existence of the spillover effect of technology among the municipalities of the region, as well as to analyze the determinants of the production value of the agricultural sector in 2006. For this purpose, we used methods derived from spatial econometric. The findings showed that in the agricultural sector of the North the labor force has a greater impact on the value of production, and that there isn't technology spillover among the municipalities. This outcome can be explained by the low-road-density in the region and by the distance between establishments, these factors don't allow the dissemination and sharing of technology. However, we verified the existence of spatial dependence in the regression disturbance term in the northern agricultural sector.

Keywords: Agriculture. Production function. Externality. Spillovers.

\section{AGROPECUARIA EN LA REGIÓN NORTE DE BRASIL: SPILLOVERS DE TECNOLOGÍA ENTRE MUNICIPIOS}

Resumen: Este trabajo tiene como objeto de estudio el sector agropecuario de la región norte del Brasil y, objetiva verificar la existencia de spillovers de tecnología entre los municipios norteño e identificar los determinantes del valor de producción de sector en el año 2006. Para alcanzar los objetivos propuestos se utilizó métodos provenientes de la econometría espacial. Los resultados encontrados muestran que en el sector agropecuario del norte brasileiro la variable mano de obra posee mayor

\footnotetext{
1 Universidade Federal de Viçosa, Departamento de Economia Rural, Viçosa, Brasil, floresreyna.edi@gmail.com, https://orcid.org/0000-0002-5626-7308

2 Universidade Federal do Acre, Departamento de Economia, Rio Branco, Brasil, rubicleis@uol.com.br, https://orcid.org/0000-0002-4556-7823

3 Universidade Federal de Viçosa, Departamento de Economia Rural, Viçosa, Brasil,
} viviani.lirio@gmail.com, https://orcid.org/0000-0001-6806-819X 
impacto sobre el valor de producción. Al mismo tiempo fue identificado que no existe spillovers de tecnología entre los municipios. Esto se explica en gran parte por la baja densidad de carreteras en la región y por la distancia entre los establecimientos. Sin embargo, fue verificado que existe dependencia espacial en el elemento del erro.

Palabras clave: Delimitar Agropecuaria. Función de producción. Externalidad. Spillovers.

\section{Introdução}

O setor agropecuário é importante para a geração de emprego e renda das sociedades e, consequentemente, para o desenvolvimento econômico dos países. No caso brasileiro, durante o milagre econômico, de 1969 a 1973, o setor agropecuário teve uma participação no Produto Interno Bruto (PIB) de 14,8\%. Mais recentemente, de acordo com dados do Banco Mundial, entre os anos de 20002016, o setor foi responsável, de forma direta, por 5,5\% do PIB mundial e por 5,5\% do PIB brasileiro (BANCO MUNDIAL, 2018).

Todavia, a perda de participação direta do setor agropecuário na economia brasileira veio acompanhada do aumento da participação indireta através de suas relações ao longo das cadeias produtivas do agronegócio, a seu montante e jusante. Esta característica fez com que o setor seja, de forma direta e indireta, responsável por 20,3\% do PIB nacional, mostrando assim, o papel estratégico da agropecuária na geração de emprego e renda dentro do país (SILVA e NONNENBERG, 2006; DIAS e OLIVEIRA, 2004).

Ainda que a agropecuária seja relevante para toda a economia do país, esta pesquisa terá apenas como objeto de estudo a região Norte. A eleição desta região ocorre em função do destaque das atividades agropecuárias para a economia nortista, bem como em virtude das diferenças, para menor, dos índices de produtividade do setor, se comparados aos de outras regiões, o que gera espaço para análises como a aqui proposta. (IBGE, 2015).

A Região Norte, formada pelos estados de Rondônia, Acre, Amazonas, Roraima, Pará, Amapá e Tocantins, possui ampla extensão territorial de 3,9 milhões de $\mathrm{km}^{2}, 42,3 \%$ do território nacional e $80 \%$ da floresta Amazônica brasileira (FREITAS, 2017). Além disso, o Norte possui população de 17,2 milhões de pessoas e caracteriza-se por apresentar altos níveis de desigualdade social, o que é posto em evidência pela presença de quantidade significativa de municípios que possuem os mais baixos indicadores socais do Brasil e que se encontram isolados geograficamente dos centros mais desenvolvidos do país. (SILVA e BACHA, 2014). 
Em contraste a essa heterogeneidade socioeconômica, a região Norte é uma das que vem apresentado maior crescimento econômico e populacional nos últimos anos. Entre 2001-2017 a população nortista cresceu 35,4\% e o PIB regional teve um incremento de 4,1\% a.a. entre 2002-2015, sendo que ambos indicadores cresceram acima da média nacional de $20,5 \%$ e $2,9 \%$ a.a., respectivamente. Porém, mesmo com este crescimento, a participação da região no PIB nacional continua sendo a menor de todas: no período de 2010 a 2013 a participação do Norte no PIB brasileiro foi de 5,4\% (IBGE, 2015; IBGE, 2018a; IBGE, 2018b; SILVA e BACHA, 2014).

Segundo os dados apresentados na Tabela 1, oriundos dos Censos Agropecuários, a agropecuária da região Norte teve mudanças expressivas entre 1995/1996, e 2006. Neste período a quantidade de estabelecimentos na região apresentou um crescimento de $6,6 \%$ e a área ocupada foi reduzida em $4,8 \%$. A redução da área ocupada no Norte é menor que a redução da mesma variável no cenário nacional, isto pode ser é explicado pelo aumento exponencial das áreas utilizadas em lavouras (156\% nas lavouras permanentes e 90,9\% nas lavouras temporárias) e pastagens (41,5\% nas pastagens plantadas), corroborando assim a importância que a pecuária e a agricultura têm para a região.

Tabela 1: Variação das principais características da agropecuária no Brasil e na região Norte durante o período de 1995/1996 a 2006.

\begin{tabular}{llrr}
\multicolumn{2}{c}{ Variáveis analisadas } & Norte (\%) & Brasil (\%) \\
\hline Estabelecimentos & & 6,63 & 6,50 \\
Área total (ha) & & $-4,84$ & $-5,64$ \\
& Lavouras permanentes & 155,98 & 54,86 \\
& Lavouras temporárias & 90,86 & 42,80 \\
Utilização de terras (ha) & $-37,63$ & $-26,16$ \\
& Pastagens naturais & 41,51 & 2,77 \\
& Pastagens plantadas & $-12,40$ & 7,21 \\
Pessoas ocupadas & Matas naturais & 0,71 & $-12,26$ \\
Números de Tratores & Matas plantadas & $-11,83$ & $-7,60$ \\
\hline
\end{tabular}

Fonte: IBGE (2017).

Por outro lado, em relação ao número de tratores, a região Norte teve um aumento de $41,5 \%$, bem acima da média nacional de 2,1\%. Considerando esta variável como uma proxy ${ }^{4}$ de modernização, poderia se dizer que houve no Norte

\footnotetext{
${ }^{4}$ Se reconhece aqui a complexidade e amplitude do conceito de modernização tecnológica, pois, este leva em consideração diferentes aspectos e indicadores do entorno social, econômico, cultural e ambiental do lugar onde são realizadas as atividades econômicas. Todavia, considerando a disponibilidade de dados e reconhecendo a existência de uma correlação entre maquinaria e produtividade, utilizou-se nesta pesquisa o número de maquinaria como proxy de modernização.
} 
um processo de modernização do setor agropecuário. Já a variável 'pessoas ocupadas' mostra que houve uma redução no número de pessoas que trabalharam no setor agropecuário.

Embora a participação da agropecuária da região Norte no PIB nacional seja a menor do país, ela apresentou crescimento nas duas principais atividades do setor entre os anos de 2010 e 2014. Na Tabela 2, os dados evidenciam que a agricultura do Norte, teve o segundo maior crescimento, de 10,3\%, entre as regiões, uma vez que o Centro-Oeste obteve um crescimento de 32,5\%. Por sua vez a pecuária nortista apresentou uma variação positiva de $8,1 \%$, ficando atrás apenas da região Sul (IBGE, 2016).

Tabela 2: Participação das grandes regiões no valor adicionado bruto por atividades2010 a 2014.

\begin{tabular}{lrrrrrr}
\hline \multirow{2}{*}{ Regiões } & \multicolumn{3}{c}{ Pecuária (\%) } & \multicolumn{3}{c}{ Agricultura (\%) } \\
& 2010 & 2014 & \multicolumn{1}{c}{$\Delta$} & 2010 & 2014 & \multicolumn{1}{c}{$\Delta$} \\
\hline Norte & 12,4 & 13,4 & 8,1 & 7,8 & 8,6 & 10,3 \\
Nordeste & 18,3 & 17,6 & $-3,8$ & 20,0 & 17,9 & $-10,5$ \\
Sudeste & 22,6 & 21,4 & $-5,3$ & 29,3 & 24,7 & $-15,7$ \\
Sul & 25,0 & 28,4 & 13,6 & 27,9 & 28,7 & 2,9 \\
Centro-Oeste & 21,7 & 19,2 & $-11,5$ & 15,1 & 20,0 & 32,5 \\
\hline
\end{tabular}

Fonte: IBGE (2016)

A partir desses dados, do potencial estratégico e do crescimento da agropecuária na região Norte, abre-se o espaço para a realização de estudos que permitam compreender sua estruturação e seu funcionamento para, dessa forma, estruturarem-se ações mais efetivas de estabilização econômica e políticas que potencializem as especificações da agropecuária nortista. Nesse intuito, este trabalho tem como problema de pesquisa investigar se a tecnologia apresenta transbordamento na produção agropecuária da região Norte e, caso este efeito exista, se haveria o melhoramento e a criação de clusters de produção do setor agropecuário que aproveitem o processo de difusão e compartilhamento de tecnologia entre produtores de municípios próximos.

O termo de transbordamento é entendido como o processo de transferência de conhecimento entre indivíduos, o qual se dá pela interação entre os agentes econômicos, potencializado pela sua proximidade geográfica. A questão geográfica torna-se relevante no quesito de transbordamento dado que permite a intensificação da comunicação entre as pessoas, e consequentemente, a difusão e adoção de novos conhecimentos. O tipo de comunicação existente entre as pessoas é 
determinado, de certo modo, por questões históricas e educacionais. Estas duas últimas características criam uma cultura comum que permite maior proximidade socioeconômica entre as pessoas (FERREIRA, 2015; GLAESER et al. 1992; BARBOSA FILHO e PESSÔA, 2010)

Vários autores tiveram como objeto de estudo a distribuição espacial da agropecuária. Em Minas Gerais, o estudo de Almeida (2005) teve como objetivo a elaboração de uma função de produção agropecuária incluindo as dimensões espaciais. Os resultados obtidos mostraram que na agropecuária mineira existe uma autocorrelação espacial presente no termo do erro. Por outro lado, Rocha e Parré (2009) procuram analisar a distribuição espacial do setor agropecuário do Rio Grande do Sul, mediante a verificação da existência de clusters. Verificou-se nesse estudo a presença de dois clusters do tipo alto-alto, e três do tipo baixo-baixo. Este mesmo estudo foi realizado para o estado de Paraná por Pinheiro, Parré e Lopes (2006), no qual se encontrou a não existência de efeitos espaciais no setor agropecuário.

Este trabalho parte da hipótese de que a produção agropecuária do Norte não apresenta dependência espacial. Isto significa que o volume de produção de uma determinada região não depende do volume de produção das regiões vizinhas. Para que exista dependência espacial, os agentes devem se encontrar geograficamente concentrados, tendo muita proximidade e acessibilidade que permitam haver compartilhamento e difusão da tecnologia. No entanto, estas peculiaridades não estão presentes na região Norte devido às características geográficas e à acentuada heterogeneidade socioeconômica. A geografia nortista e a baixa densidade rodoviária dificultam o transporte e a heterogeneidade não permite a criação de uma cultura comum entre os indivíduos que permita uma boa difusão de informação. (SANTOS, 2014; SILVA e BACHA, 2014; BARBOSA FILHO e PESSÔA, 2010).

$O$ objetivo geral deste trabalho é verificar a existência do efeito transbordamento de tecnologia no setor agropecuário nos municípios da região Norte em 2006. Especificamente, procura-se: a) identificar possíveis agrupamentos espaciais de transbordamento tecnológicos entre os municípios da região Norte e; b) determinar a magnitude do transbordamento tecnológico agropecuário sobre os municípios.

A relevância dos estudos no setor agropecuário está relacionada com a elaboração de um conhecimento sistemático e teórico que permita um diagnóstico adequado sobre a estruturação, organização, funcionamento e dinâmica do setor. 
Dessa forma, com ajuda destes conhecimentos, a elaboração de políticas de desenvolvimento rural e intervenções dos formuladores de políticas agrárias seriam mais eficientes. (MAZOYER E ROUDART, 2010).

Para a criação desse conhecimento sistemático da agropecuária na região Norte foram incorporadas à pesquisa, além dos elementos comuns de uma função de produção, as variáveis com dimensões espaciais que permitiram captar as associações e o grau de interação dos municípios no sistema agropecuário, também conhecido na geografia econômica, como o efeito transbordamento.

Além da parte introdutória, o presente estudo está dividido em mais cinco seções. A próxima seção apresenta dois conceitos importantes que darão suporte a este trabalho. Na terceira seção se apresenta a metodologia, logo em seguida, na quarta seção, se mostram os principais resultados da pesquisa e, na última seção, são apresentadas as considerações finais.

\section{Referencial Teórico}

O arcabouço teórico deste trabalho tem como alicerces dois conceitos microeconômicos que serão apresentados a seguir:

\section{Função de Produção}

A análise da agropecuária nortista será baseada, primeiramente, no arcabouço teórico da Teoria de Produção que permite a elaboração de uma função de produção agropecuária. Esta é definida como a relação que indica a quantidade que se pode obter de um produto a partir da combinação de determinada quantidade de fatores de produção (VARIAN, 2000).

Esta função pode ser representada da seguinte maneira:

$$
Q=f(k, t, m)
$$

Onde:

Q: representa o valor médio de produção agropecuária;

k: o capital necessário para a produção;

$t$ : representa a terra utilizada

$m$ : representa a força de trabalho

A função de produção escolhida neste trabalho será do tipo Cobb-Douglas, isto devido a "(...) sua facilidade de interpretação dos resultados e as boas qualidades estatísticas dessa especificação, via de regra, em termos de aderências aos dados observados" (CASTRO, 2002, p. 95). 
A agropecuária, na sua essência, é uma atividade que depende tanto de variáveis endógenas quanto exógenas para explicar o comportamento de seu volume de produção. Portanto, a equação comportamental da função de produção deste trabalho deverá incluir variáveis com dimensões espaciais que permitam captar, caso existam, as associações e interação dos agentes econômicos do sistema agrário do Norte (ALMEIDA, 2005).

\section{Externalidades}

Para analisar os efeitos de transbordamento, que podem existir no setor agropecuário da região Norte, se levará em consideração o conceito de externalidades. Varian (2006) define externalidades como bens que não são comercializados no mercado e que provocam impactos positivos ou negativos nos agentes econômicos. Esta falha de mercado causa alocações ineficientes dos recursos no sentido de Pareto.

Mankiw (2012), por sua vez, diz que as externalidades são os efeitos não compensados que determinadas ações dos agentes têm sobre o bem-estar dos outros. Estas externalidades podem ser negativas ou positivas, dependendo do tipo de efeito que eles causem sobre os demais agentes. Se a ação de um agente impõe custos ao consumo ou produção a outros, isto significa que existe externalidade negativa. Em contraste, se a ação do mesmo agente traz benefícios para outros indivíduos, se tem uma externalidade positiva.

Varian (2006) também menciona os conceitos de externalidade na produção e no consumo. Esta está relacionado com a preocupação que um consumidor tem frente às ações de outro agente econômico. A externalidade na produção surge quando a função de custos de uma determinada empresa é influenciada pelas ações ou escolhas de terceiros (consumidor ou empresa), prejudicando ou beneficiando de forma direta as possibilidades de produção da mesma.

O conceito de externalidade na produção será utilizado ao longo do trabalho, pois com ele é possível identificar o tipo e nível de impacto que determinado município possui sobre o volume de produção do setor agropecuário dos seus vizinhos. Nesse sentido, os custos ou benefícios gerados por um município serão incluídos nas funções de produção dos outros que ficam perto dele.

A importância deste conceito na análise da agropecuária deve-se, segundo Almeida (2005), ao fato do setor apresentar grandes dependências entre as regiões vizinhas. Isto acontece porque o agricultor tem facilidade de observar seus vizinhos 
e, também a agricultura possui uma distribuição no território nacional associada a variáveis culturais, ambientais e climatológicas. Estas características permitem a existência de aglomerações (clusters) naturais relativos à localização da matéria prima.

Além disso, a concentração de atividades econômicas num determinado espaço pode causar impactos positivos ou negativos no dinamismo econômico das regiões. Sendo assim, a existência de aglomeração de municípios da região Norte pode trazer efeitos de transbordamentos de tecnologia no valor de produção do setor agropecuário.

\section{Metodologia}

\section{Análise Exploratória de Dados Espaciais.}

O método de Análise Exploratória de Dados Espaciais (AEDE) é um conjunto de técnicas de análise de dependência e heterogeneidade espacial que visa descrever e/ou identificar a distribuição espacial dos dados, os clusters e os outliers espaciais (ALMEIDA, 2012),

É importante salientar que para este tipo de análise, a forma mais adequada de identificar o fenômeno espacial é através da utilização de variáveis intensivas ou per capita, portanto, todas as variáveis utilizadas no trabalho serão dividas pelo número de estabelecimento presentes nos municípios. Almeida (2005, p. 3) destaca que: “... a sua importância reside no fato de que essas variáveis levariam em conta externalidades relevantes na consideração do processo produtivo, tais como efeitos de aglomeração e/ou congestão". Permitindo assim, conseguir o efeito de transbordamento da tecnologia de produção na agropecuária nortista.

A identificação dos fenômenos espaciais nos dados demanda a escolha de uma matriz de ponderação espacial $\left(w_{i j}\right)$ que dê suporte à AEDE. Esta matriz reflete as interações ou conexões espaciais entre as regiões, seguindo critérios de proximidade. Os critérios podem ser de contiguidade ou distância geográfica.

O processo de escolha da matriz $w$ é de suma importância porque é ela que determinará a construção das defasagens espaciais. Caso as defasagens espaciais não sejam medidas corretamente, os resíduos do modelo continuarão mostrando dependência espacial e, portanto, o modelo de regressão espacial será viesado e inconsistente (ALMEIDA, 2012). 


\section{Autocorrelação Espacial Global}

O teste mais comum utilizado para identificar autocorrelação espacial global foi desenvolvido pelo estatístico Moran em 1948 e é conhecido como I de Moran. Este proporciona o grau de associação e interação espacial entre regiões vizinhas, mostrando como valores de determinada variável em análise em uma região podem ser afetados por valores da mesma variável em regiões vizinhas (ANSELIN, 2003)

O I de Moran é um coeficiente de autocorrelação que trabalha sob a hipótese nula de aleatoriedade espacial. Esta hipótese é cumprida quando o valor obtido da estatística I de Moran é igual ao seu valor esperado de $E(I)=-1 /(n-1)$. Valores de I que sejam maiores (menores) ao seu valor esperado evidenciaram existência de autocorrelação espacial positiva (negativa) (ALMEIDA, 2012; ANSELIN e BERA 1998). A fórmula do teste I de Moran é expressa da seguinte maneira:

$$
I_{t}=\left(\frac{N}{S_{0}}\right)\left(\frac{\theta / W_{B}}{\theta_{\theta}}\right)
$$

Onde $N$ representa o número de observações; $e=Y-X B$ é o vetor dos resíduos do MQO (Mínimos Quadrados Ordinários); $W$ é matriz de pesos espaciais e; $S_{0}$ é o escalar padronizado igual à soma de todos os elementos da matriz W (ANSELIN e BERA, 1998)

Outra forma de interpretar a estatística I de Moran é por meio do diagrama de dispersão de Moran. Este está dividido em quatro quadrantes, que correspondem a padrões de associação linear espacial que determinada região tem com suas vizinhas. Estas associações ou clusters podem ser do tipo AA (alto- alto), BB (baixo-baixo), AB (alto-baixo) e BA (baixo-alto). Os clusters AA e BB possuem autocorrelação positiva e os clusters AB e BA autocorrelação negativa (ALMEIDA, 2012).

O teste I de Moran também pode ser usado num contexto bivariado, ou seja, para identificar ou descobrir se os valores de duas variáveis diferentes guardam alguma relação entre as regiões analisadas. Este coeficiente é calculado por:

$$
I^{\theta_{1} \theta_{2}}=\left(\frac{N}{S_{0}}\right)\left(\frac{\theta_{1} W e_{2}}{\theta_{1} \theta_{2}}\right)
$$

Embora a análises de autocorrelação espacial global mostre certa evidência da existência ou não do fenômeno espacial, ela, de per si, não é suficiente para um bom estudo da distribuição espacial de dados. Nesse sentido, é importante fazer uma análise de cada região para identificar possíveis autocorrelações espaciais locais. 


\title{
Autocorrelação Espacial Local
}

Uma análise apenas global das informações pode ocultar padrões de autocorrelação espacial local, provocando umas das três situações mencionadas a seguir:

\begin{abstract}
A primeira envolve a indicação de um I de Moran global insignificante, porém, pode haver indicações de autocorrelação espacial local insignificante, positiva ou negativa. A segunda situação implica uma indicação positiva do I de Moran global, que oculta autocorrelação espacial local negativa insignificante. A terceira situação denota que a evidência de uma autocorrelação espacial global negativa pode acomodar indícios de autocorrelação espacial local positiva para certos grupos dos dados (PEROBELLI et al. 2005, p. 5)
\end{abstract}

Para solucionar o problema presente na determinação de autocorrelação espacial global, se faz uso de um indicador proposto por Anselin em 1995, chamando de Local Indicator of Spatial Association (LISA). Este indicador permite identificar os padrões de aglomeração espacial por meio da decomposição de indicadores, como o I de Moran, na contribuição que cada observação individual possui. (ANSELIN, 1995).

O indicador tipo LISA utilizado neste trabalho é o coeficiente I de Moran local expresso da seguinte maneira:

$$
I_{t}=z_{i} \sum_{j=1}^{j} w_{i j} z_{j}
$$

Os resultados obtidos no cômputo de lt são apresentados em formas de mapas. Estes combinam os níveis de significância de cada observação e as informações do diagrama de dispersão de Moran.

\section{Modelo Econométrico Espacial}

Considera-se como modelo econométrico espacial aquele que incorpora, dentro do modelo clássico de regressão linear (MCRL), as defasagens espaciais com a finalidade de controlar a dependência espacial do fenômeno. As defasagens podem estar presentes na forma de variável dependente $(\mathrm{W} y)$, variável

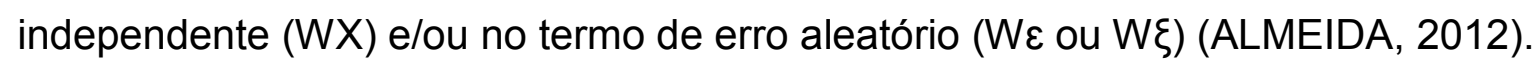

Todos os modelos utilizados na econometria espacial derivam de um único modelo conhecido como Modelo de Dependência Espacial Geral (GSM) conforme se observa na equação 5. A partir dele se encontra o modelo mais adequado para controlar os aspectos espaciais do fenômeno estudado neste trabalho.

O modelo GSM é representado pela seguinte fórmula: 


$$
\begin{aligned}
& y=\rho W_{1} y+X \beta+W_{1} X \tau+\xi \\
& \xi=\lambda W_{2} \xi+\varepsilon \text { ou } \xi=\gamma W_{1} \varepsilon+\varepsilon ; \operatorname{ondes}\left(0, \sigma^{2} I\right)
\end{aligned}
$$

Onde $y$ é o vetor das observações da variável dependente; $\beta$ é o vetor de coeficientes a serem estimados; $\mathrm{X}$ é a matriz das variáveis explanatórias; $W_{1} y$ é 0 vetor da variável dependente defasada e $\rho$ é seu parâmetro autorregressivo; $\tau$ é um vetor de coeficientes dos impactos das externalidades que acompanha a matriz que representa a defasagem espacial das variáveis explicativas $W_{1} X ; \xi$ representa 0 termo de erro auto correlacionado que está composto pelo parâmetro autorregressivo espacial do erro ( $\lambda$ ou $\gamma$ ), pela defasagem do termo de erro $\left(W_{2} \xi\right.$ ou $\left.W_{1} \varepsilon\right)$ e pelo vetor de erros com variância constante e média zero $(\varepsilon) ; W_{1} \mathrm{e}$ $W_{2}$ são matrizes de ponderação espacial. Os parâmetros autorregressivos $(\lambda, \gamma, \rho)$ e o vetor $\tau$ adotam valores apenas entre -1 e 1 .

De acordo com os valores obtidos pelos parâmetros autorregressivos são obtidos os seguintes modelos:

[...] o modelo de defasagem espacial (SAR) [...], emerge se impuser as restrições sobre os escalares espacial $\gamma=0, \lambda=0$ e o vetor $\mathrm{T}=0$, bem como $\rho$ $\neq 0$. $O$ modelo de erro autorregressivo espacial (SEM) [...], envolve impor as restrições $\rho=0, \gamma=0$ e o vetor $\mathrm{T}=0$, assim como $\lambda \neq 0$. O modelo de erro de média móvel espacial (SMA) implica colocar as restrições $\rho=0, \lambda=0$, o vetor $\mathrm{T}=0$ e o escalar $\mathrm{\gamma \neq 0}$. Já o modelo de Durbin espacial (SDM), [...] implica que se imponham as restrições de que $\rho \neq 0$ e o vetor $\mathrm{T} \neq 0$, ao passo que $\lambda=0$ e $\mathrm{Y}=0[\ldots]$ (ALMEIDA, 2012, p. 179).

Outros modelos que também podem ser identificados a partir do GSM são: o modelo autorregressivo-média móvel espacial (SARMA) emerge quando se obtém que $\rho \neq 0$ e $\lambda \neq 0$; o modelo regressivo cruzado espacial (SLX) surge quando apenas 0 vetor é $\mathrm{T} \neq 0$.

Os métodos que são usados para estimar os modelos econométricos espaciais supracitados, dependem do cumprimento ou não do pressuposto de normalidade do erro. Assim, se o pressuposto for satisfeito, o modelo é estimado pelo método de Máxima Verossimilhança (MV), caso contrário e dependendo do modelo obtido, se utiliza um dos métodos a seguir: Método de Quase Máxima Verossimilhança (QMV); Variáveis Instrumentais (VI), Método Generalizado dos Momentos (MGM) e; Mínimo Quadrado de dois Estágios (MQ2E) Espacial Generalizado.

Todavia, caso não seja identificado nenhum modelo de econometria espacial, a produção da agropecuária do Norte será analisada através do método da econometria convencional de mínimo quadrados ordinários (MQO). 
Por outro lado, na presença de aspectos espaciais nos dados analisados, o coeficiente convencional R-quadrado deixa de ser um indicador adequado para determinar a qualidade de ajuste do modelo. Portanto, a avaliação e escolha do modelo espacial está guiado pelos critérios de informação Akaike (AIC) e de Schwartz (SC).

\section{Fontes de Dados}

Os dados utilizados neste trabalho são secundários e foram obtidos do censo agropecuário de 2006, divulgado pelo Instituto Brasileiro de Geografia e Estatística (IBGE). Os dados terem são do tipo cross section.

Seguindo com a teoria sobre a identificação dos fenômenos espaciais, todas as variáveis utilizadas neste trabalho são intensivas. Nesse sentido, elas foram divididas pelo número de estabelecimentos presentes em cada município.

A seguir, no Quadro 1, apresenta-se as variáveis utilizadas na análise do setor agropecuário dos municípios da região Norte.

Quadro 1: Sínteses das variáveis utilizadas na pesquisa

\begin{tabular}{|c|l|c|c|}
\hline Variável & \multicolumn{1}{|c|}{ Descrição } & $\begin{array}{c}\text { Sinal } \\
\text { esperado }\end{array}$ & Referencial teórico \\
\hline vbp & $\begin{array}{l}\text { Valor médio da produção (por } \\
\text { estabelecimentos) da agropecuária } \\
\text { dos municípios da região Norte } \\
\text { durante o período de agosto de } \\
\text { 2005 e julho de 2006 }\end{array}$ & $\begin{array}{c}\text { Almeida (2004) e } \\
\text { Almeida (2012). }\end{array}$ \\
\hline A & $\begin{array}{l}\text { Área do estabelecimento } \\
\text { agropecuário em 2006 nos } \\
\text { municípios da região Norte }\end{array}$ & + & $\begin{array}{l}\text { Almeida (2004) e } \\
\text { Almeida (2012). }\end{array}$ \\
\hline Mb & $\begin{array}{l}\text { Número de pessoas ocupadas por } \\
\text { estabelecimento. }\end{array}$ & + & $\begin{array}{c}\text { Almeida (2004) e } \\
\text { Almeida (2012). }\end{array}$ \\
\hline kfísico & $\begin{array}{l}\text { Número de máquinas de colheita, } \\
\text { de plantio, tratores, caminhões por } \\
\text { estabelecimento. (proxy do nível } \\
\text { tecnológico) }\end{array}$ & + & $\begin{array}{c}\text { Almeida (2004) e } \\
\text { Almeida (2012). }\end{array}$ \\
\hline Invest & $\begin{array}{l}\text { Total de investimento em reais por } \\
\text { estabelecimento }\end{array}$ & + & Botti (2014) \\
\hline
\end{tabular}

Fonte: elaboração do autor. 


\section{Resultados e Discussões.}

\section{Agrupamentos espaciais de tecnologia agropecuária.}

Antes de analisar a existência de autocorrelação espacial na agropecuária da região Norte, observa-se o desempenho da produtividade do setor em todos os municípios da região para 2006. A Figura 1 mostra que a distribuição espacial da produtividade dos municípios apresenta características não aleatórias, podendo concluir de forma intuitiva que existe concentração de municípios com produtividade semelhante.

Todavia, a concentração dos municípios com produtividade semelhante não é um indício de dependência espacial, pois tal como afirma Almeida (2005), diagnósticos produtos da simples visualização podem gerar conclusões sobre padrões espaciais que podem ou não existir. Por sua vez Kahneman (2012) diz que elaborar afirmações apenas com a intuição produzem diagnósticos estatísticos viesados.

Contudo, na Figura 1 é possível verificar que a maioria dos municípios da região Norte possui baixa produtividade. Isto fica mais evidente quando se analisa apenas os estados de Amazonas, Acre, Amapá, Rondônia e Roraima. Já no Pará e em Tocantins observa-se diversidade no valor médio da produção nas mesorregiões Oriental de Tocantins e Sudeste paraense.

Ainda com base na Figura 1, se obtém informação sobre a presença de outliers $^{5}$ globais, pois verifica-se a existência de dois municípios com valores discrepantes na variável analisada. Estes são: Pacaraima e Ulianópolis, localizados nos estados de Roraima e Pará respectivamente.

Conforme o IBGE (2017), a participação da agropecuária no PIB municipal de Pacaraima é de apenas 17,3\%, ficando atrás do setor de serviços (66,9\%). Este nível de participação da agropecuária não é expressivo para refletir o comportamento observado na Figura 1. O valor discrepante na produtividade do município se explica principalmente pelo grau de concentração de renda do setor apenas 31 estabelecimentos produzem o total de valor bruto da produção gerado pelo setor- e pelo alto nível de capital físico (tecnologia) que ele possui (IBGE, 2009).

\footnotetext{
${ }^{5}$ Além dos dois municípios que foram considerados como outliers globais, também foram identificados outros dois municípios que apresentaram valor médio de produção agropecuária elevado. Os estabelecimentos dos municípios de Campos Lindos (TO) e São Miguel do Guamá (PA) tiveram um valor de produção médio de $\mathrm{R} \$$. 235701.01.
} 


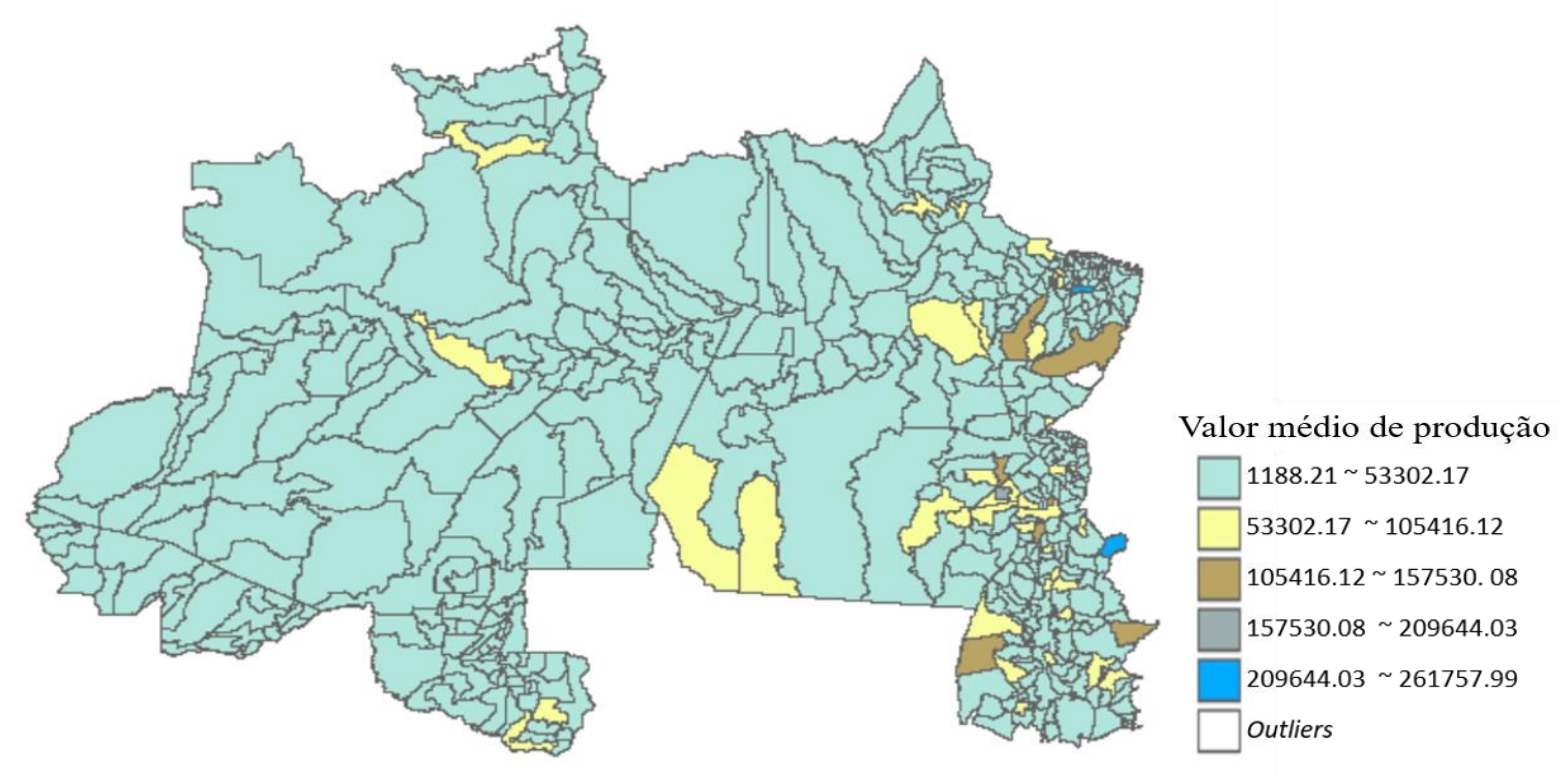

Figura 1: Valor médio de produção por estabelecimentos da agropecuária nos municípios da região Norte em 2006 em reais.

Fonte: elaboração do autor.

Ulianópolis, por sua vez, é um município completamente agrário; $73,5 \%$ do PIB municipal provêm da agropecuária. Isto, conjuntamente com a quantidade de capital físico e o investimento por estabelecimento, explica o valor discrepante na produtividade que o município apresenta na Figura 1.

A identificação de existência de autocorrelação espacial iniciou-se com a aplicação de teste global I de Moran. Na tabela 3 são apresentados os coeficientes do teste para quatros diferentes matrizes de peso espaciais referente ao valor de produção dos municípios. Mostra-se, a um nível de significância de 1\%, um nível estatisticamente significativo de dependência espacial positiva entre os municípios da região para todas as matrizes. Isto significa que municípios com valores de produção altos (baixos) estão cercados por vizinhos com a mesma característica.

Para a realização dos próximos testes e na estimação do modelo econométrico que melhor capture o comportamento do fenômeno da agropecuária nortista é considerada a matriz de peso espacial que apresentou maior coeficiente de I de Moran. Sendo assim, a matriz de peso 4 vizinhos mais próximos (4 vizinhos) foi escolhida.

Tabela 3: Coeficiente de I de Moran univariada para o logaritmo do valor médio da produção dos municípios da região Norte em 2005/2006 


\begin{tabular}{lcc} 
Matrizes & I de Moran & p-valor \\
\hline Rainha & 0,2213 & $0,0000^{\star \star \star}$ \\
Torre & 0,2255 & $0,0000^{\star \star \star}$ \\
4 vizinhos & 0,2594 & $0,0000^{\star \star \star}$ \\
5 vizinhos & 0,2482 & $0,0000^{\star \star \star}$ \\
\hline
\end{tabular}

Fonte: elaboração do autor

Obs.: ${ }^{* \star *}$ significativos a 1\%; 99999 permutações aleatórias.

Nesse sentido, conforme a tabela 4, temos que a um nível de significância de $1 \%$, todas as variáveis estudadas neste trabalho apresentam coeficientes I de Moran univariado positivos, ou seja, municípios com determinada característica estão rodeados por vizinhos que são semelhantes a eles.

Nas análises do coeficiente I de Moran bivariado, observa-se que existe autocorrelação espacial positiva entre as variáveis capital físico e investimento e a variável dependente. Dessa forma, se afirma que os municípios com valor médio de produção alto, estão cercados por municípios que apresentam as seguintes características: estabelecimentos com grande extensão de terra, alto nível de investimento e alto grau de tecnologia.

Tabela 4: Coeficiente I de Moran bivariado entre o logaritmo do valor médio de produção e as outras variáveis estudadas referentes ao setor agropecuário da região norte em 2006

\begin{tabular}{ccccc}
\hline Variável & $\begin{array}{c}\text { I de Moran } \\
\text { univariado }\end{array}$ & p-valor & $\begin{array}{c}\text { I de Moran } \\
\text { bivariado }\end{array}$ & p-valor \\
\hline Ln_A & 0,5923 & $0,0000^{* * *}$ & 0,2566 & $0,0000^{* * *}$ \\
Ln_Mb & 0,2138 & $0,0000^{\star * *}$ & $-0,0662$ & $0,0023^{* * *}$ \\
Ln_kfísico & 0,5265 & $0,0000^{\star * *}$ & 0,2456 & $0,0000^{\star * *}$ \\
Ln_invest & 0,3683 & $0,0000^{* * *}$ & 0,2756 & $0,0000^{* * *}$ \\
\hline
\end{tabular}

Fonte: elaboração do autor

Obs.: significativo a 1\%; 99999 permutações aleatórias.

Por outro lado, as análises do coeficiente bivariado entre a quantidade de mão-de-obra e valor de produção mostra uma relação negativa, ou seja, municípios com alto valor de produção estão circunvizinhados por municípios com pouca quantidade de mão-de-obra. Para os municípios com baixos níveis de produção a interpretação do coeficiente é feita de forma oposta.

Como já foi mencionado anteriormente, o teste global de I de Moran pode ocultar padrões de associações locais. Para evitar isso, em seguida é apresentado o indicador LISA.

$\mathrm{Na}$ Figura 2 é possível verificar a existência de 4 tipos de clusters com os níveis de significância de $5 \%$ e $1 \%$. Estes clusters estão distribuídos em todo o 
território da região e classificam-se como alto-alto $(A A)$, baixo-baixo $(B B)$, alto-baixo (AB) e baixo-alto (BA).

Os clusters do tipo AA são formados por municípios que possuem um alto valor médio de produção, rodeados por municípios com essa mesma característica. Conforme a Figura 2, as aglomerações com esta classificação estão localizadas nos estados: Pará, Roraima, Rondônia e Tocantins, mais especificamente, nas mesorregiões Norte de Roraima, Sudeste Paraense, Leste Rondoniense e Ocidental do Tocantins.

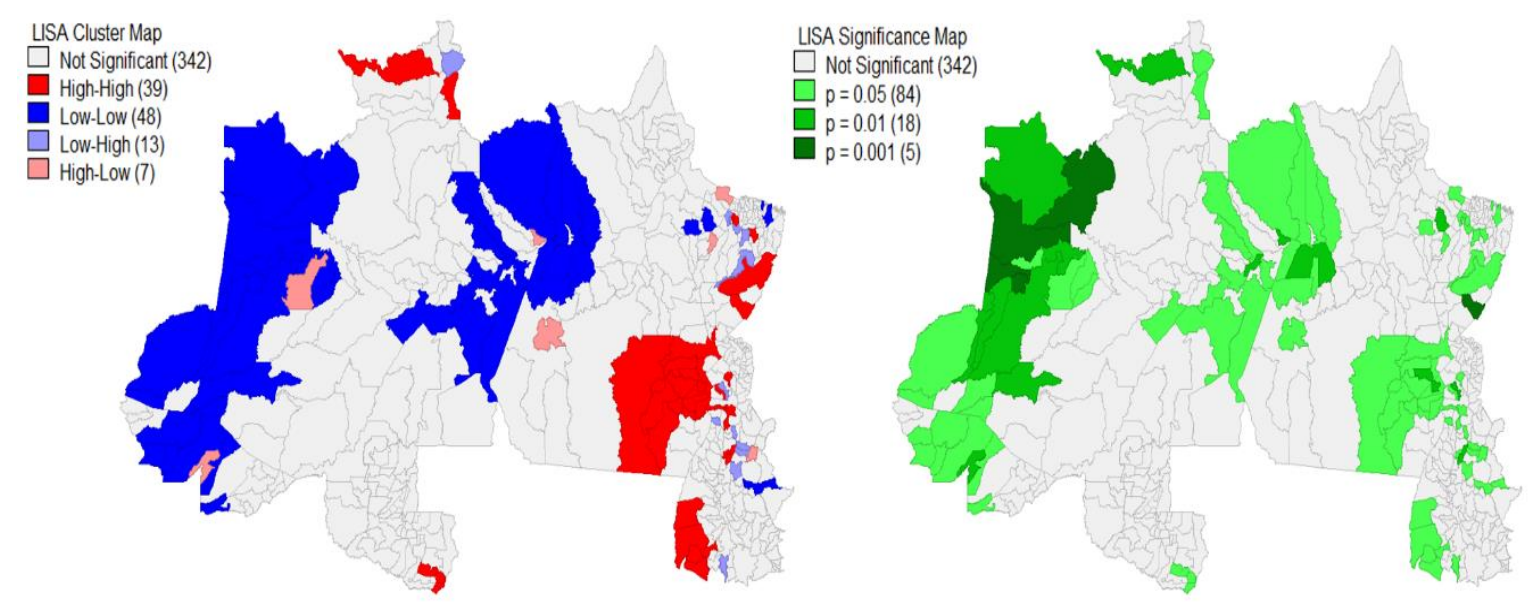

Figura 2: Mapa de cluster e nível de significância do logaritmo do valor médio de produção na região norte em 2006.

Fonte: elaboração do autor

Por outo lado, os clusters do tipo $A B$ são formados por municípios que possuem estabelecimentos com um alto valor médio de produção, porém os seus vizinhos apresentam baixo valor médio de produção. Os agrupamentos deste tipo se encontram localizados nas mesorregiões: Baixo Amazonas (PA), Oriental Tocantins (TO), Vale do Acre (AC), Centro Amazonense (AM), Marajo e Nordeste Paraense (PA).

O terceiro tipo de clusters identificado pelo LISA é do tipo BA. Neste se encontram os municípios com baixo nível de produção que estão cercados por vizinhos com a característica oposta, ou seja, altos valores de produção. Estes clusters se localizam nas mesorregiões Norte de Roraima (RO), Oriental e Ocidental de Tocantins (TO), Nordeste Paraense (PA).

Por último, o tipo de clusters com maior presença nesta análise é o BB. Isto significa que municípios com baixo nível de produção agropecuária têm como vizinhos municípios com o mesmo nível de produção. Em conformidade com a 
Figura 2, as aglomerações deste tipo estão localizadas principalmente nos estados do Amazonas, Acre e parte do Pará.

A explicação deste fenômeno relaciona-se com o baixo nível estadual de modernização do setor agropecuário nas mesorregiões onde existem os clusters BB. Pois os clusters presente nas mesorregiões Sudoeste, Centro e Norte amazonense (AM), Baixo Amazonas (PA), no Vale do Juruá e no Vale do Acre (AC), são formados por municípios que possuem pouco ou quase nenhum capital físico (kfísico) nos estabelecimentos (IBGE, 2009; SILVA e FERNANDES, 2005).

Finalizando a aplicação dos testes gerais se obteve indícios de presença de dependência espacial na distribuição do valor médio de produção da agropecuária nos municípios da região Norte. Todavia, ainda há necessidade de realizar os testes específicos para identificar onde acontecem os processos de autocorrelação espacial para ratificar ou não os resultados obtidos pelo coeficiente de I de Moran e 0 indicador LISA.

\section{Efeito transbordamento no setor agropecuário entre os municípios}

A seguir são expostos os resultados do modelo que melhor capture 0 comportamento do setor agropecuário da região Norte. A escolha desse modelo inicia-se a partir da estimação de um modelo clássico de regressão linear (MCLR) que mostre um diagnóstico preliminar da função de produção do setor.

A tabela 5 traz os resultados do MCRL estimados por MQO. A um nível de significância de $1 \%$ e $5 \%$, os estimadores obtidos estão em consonância com os sinais esperados para as variáveis explicativas. Dessa maneira, se pode concluir que uma variação relativa positiva das variáveis independentes provoca um aumento relativo no valor médio de produção dos municípios.

Tabela 5: Resultados da regressão do MCLR por MQO para o logaritmo natural do valor médio de produção (In_vpb) do setor agropecuário da região norte em 2006

\begin{tabular}{|c|c|c|c|c|}
\hline Variáveis & Coeficiente & Erro-padrão & t-student & $p$-valor \\
\hline constante & 6,2584 & 0,3782 & 16,5461 & $0,0000^{\star \star \star}$ \\
\hline In_a & 0,2867 & 0,0402 & 7,1233 & $0,0000^{* * *}$ \\
\hline In_mb & 0,6562 & 0,1771 & 3,7052 & $0,0002^{\star \star \star}$ \\
\hline In_kfísico & 0,2570 & 0,0500 & 5,1390 & $0,0000^{\star * *}$ \\
\hline In_invest & 0,1009 & 0,0451 & 2,2369 & $0,0258^{* \star}$ \\
\hline $\mathrm{R}^{2}$ & 0,4137 & & Jarque-Bera & $0,0000^{\star \star \star *}$ \\
\hline F-statistic & 78,3388 & & Breush-Pagan & $0,0276^{\star *}$ \\
\hline Prob (F-statistic) & $0,0000^{* \star *}$ & & White & $0,0000^{\star \star *}$ \\
\hline condition number & 21,910 & & & \\
\hline
\end{tabular}


Obs.: ${ }^{* * *}$ significativo a $1 \% \mathrm{e}^{* *}$ significativo a $5 \%$

Quando se analisa o teste $\mathrm{R}^{2}$ se obtém que as variações relativas do valor médio de produção são explicadas em 41,4\% pelas variáveis explanatórias escolhidas para este estudo. Observando o teste de multicolinearidade percebe-se que o modelo não apresenta este problema, pois o valor do condition number é menor que 30. O teste normalidade (Jarque-Bera) e de heterocedasticidade (BreushPagan e White) indicam que os erros não possuem uma distribuição normal e o modelo apresenta problemas de heterocedasticidade.

Após estimar o modelo clássico e observar os problemas nele contido, foi necessário fazer o diagnóstico de dependência espacial utilizando os testes específicos. Pela observação da tabela 6 se revela que os testes de Multiplicadores de Lagrange, tanto na defasagem da variável dependente como no erro, se mostram significativos, portanto, rejeita-se a hipótese nula de distribuição aleatória do valor de produção agropecuário. Com as versões robustas dos mesmos testes, temos que esta dependência espacial é determinada no termo de erro. O teste AnselinKelejian, a um nível de significância de 5\%, comprova novamente a existência do fenômeno espacial nos municípios da região.

Tabela 6: Diagnóstico de dependência espacial- teste específicos

\begin{tabular}{lcc}
\hline \multicolumn{1}{c}{ Teste } & Valor & p-valor \\
\hline Anselin-Kelejian & 5,341 & $0,0208^{\star *}$ \\
$M L \lambda$ (erro) & 11,472 & $0,0007^{\star * \star}$ \\
$M^{*} \lambda$ (erro robusto) & 7,588 & $0,0059^{\star * *}$ \\
$M L \rho$ (defasagem) & 18,901 & $0,0000^{\star * *}$ \\
$M^{*} \rho$ (defasagem robusta) & 0,159 & $0,6906^{\text {NS }}$ \\
\hline
\end{tabular}

Fonte: elaboração do autor

Obs.: ${ }^{* * *}$ significativo a $1 \%,{ }^{* *}$ significativo a $5 \%$, e ${ }^{\text {NS }}$ não significativo.

Sendo assim, o próximo passo é estimar a função de agropecuária nortista com os GSM, SARMA e SAM, para validar os testes específicos apresentados na tabela 6. No modelo GSM foi incluído a defasagem espacial da variável referente ao logaritmo natural do capital físico, pois um dos objetivos deste trabalho é verificar se a tecnologia apresenta efeito de transbordamento.

Devido a não normalidade dos erros, os modelos supracitados, e de acordo com a recomendação de Anselin (2003), foram estimados pelo Método de Momentos Generalizados (GMM). Os resultados encontrados com ajuda do software GeodaSpace 1.0 e o Geoda 1.12 são apresentados na tabela 7. 
Almeida (2005) diz que numa função do tipo Cobb-Douglas a constantes das regressões representaria 0 parâmetro de eficiência comum entre todos os municípios presente numa determinada região. Considerando tanto o MCRL como as regressões (2) e (3), estas últimas presentes na tabela 7, observa-se que o valor deste parâmetro de eficiência é estável.

Já as variáveis explicativas que não incluem a dimensão espacial, apresentaram os mesmos sinais que no modelo clássico e todas foram significativas a $1 \%$ e $5 \%$. Por sua vez, a variável de interesse que definiria a existência ou não de transbordamento de tecnologia mostrou-se não significativa na regressão (1). A não significância desta variável valida a hipótese levantada na pesquisa, portanto, a falta de acessibilidade - caracterizada pela baixa densidade rodoviária - e as grandes distâncias entre os estabelecimentos - devido a extensão da região norte - dificultam ou minimizam o compartilhamento de informação e tecnologia entre eles.

Tabela 7: Resultados das modelos econométricos espaciais do valor de produção do setor agropecuário da região norte em 2006.

\begin{tabular}{cccc}
\hline Variáveis & GERAL (1) & SARMA (2) & SMA (3) \\
\hline constante & 8,9350 & 6,8465 & 6,2995 \\
In_a & $(0,0022)$ & $(0,0000)$ & $(0,0000)$ \\
In_mb & 0,3647 & 0,3303 & 0,3216 \\
& $(0,0000)$ & $(0,0000)$ & $(0,0000)$ \\
In_kfísico & 0,6197 & 0,5875 & 0,6052 \\
& $(0,0006)$ & $(0,0017)$ & $(0,0007)$ \\
In_invest & 0,2495 & 0,2480 & 0,2431 \\
& $(0,0000)$ & $(0,0000)$ & $(0,0000)$ \\
w_Infisico & 0,0958 & 0,0876 & 0,0880 \\
& $(0,0134)$ & $(0,0254)$ & $(0,0269)$ \\
P & 0,1854 & - & - \\
& $(0,3210)$ & & - \\
Y & $-0,3415$ & $-0,0584$ & \\
Pseudo_R & $(0,2907)$ & $(0,6335)$ & 0,2880 \\
Akaike & 0,5166 & 0,3489 & $(0,0000)$ \\
Schwartz & $(0,0000)$ & $(0,0017)$ & 0,4125 \\
\hline
\end{tabular}

Fonte: Elaboração do autor.

Obs.: os valores nos parênteses representam o $p$-valor dos coeficientes.

Além disso, deve-se considerar a diversidade cultural e diferenças sociais entre os municípios nortistas, assim como também, o baixo nível de instrução dos produtores, pois aproximadamente $94 \%$ deles possuem no máximo ensino fundamental completo (IBGE, 2018c). Embora, o nível educacional dos produtores 
não seja uma variável que explique na sua totalidade o grau de modernização tecnológica das atividades agrícolas e pecuárias, ela é uma ferramenta que permite, de acordo com Barbosa Filho e Pessôa (2010), criar uma cultura comum entre os indivíduos de uma sociedade. Esta cultura comum gera maior proximidade entre as pessoas, facilitando a comunicação e difusão de diversos tipos de conhecimentos, entre eles o tecnológico.

A defasagem espacial do valor de produção da região Norte $(\rho)$ também foi não significativo, tanto na regressão (1) como na (2). Do total de estabelecimentos computados no censo para a região, IBGE (2018c), 86,7\% foram classificados como agricultura familiar. Estes foram responsáveis por $55,5 \%$ do valor total de produção, os quais provieram principalmente de atividades agrícolas. Já os outros estabelecimentos foram responsáveis de $44,5 \%$ do valor de produção total, do quais $61,4 \%$ foram produzidos pela pecuária. É importante salientar também que no Norte atividades relacionadas à pecuária bovina e criação de outros animais utilizavam, em 2006, 65,9\% do total de maquinas existentes na região. Portanto, infere-se que a explicação para não existir dependência espacial nesta variável, além da heterogeneidade socioeconômica, seria a grande representatividade da agricultura familiar existente na maioria dos estados da região Norte, que contam com baixo nível de tecnologia.

Pelos critérios de Akaike e Schwartz o modelo que melhor se ajusta aos dados é o modelo SMA, pois apresentou o menor valor. O SMA define que a dependência espacial do setor agropecuário do Norte está presente no termo de erro.

Toda análise feita dos coeficientes aqui neste trabalho, foi a partir da regressão (3). Sendo assim, a variação de $1 \%$ na área dos estabelecimentos provocaria uma variação média diretamente proporcional no valor de produção de 0,32\%. Já com relação a capital físico, se obteve que o aumento (redução) de $1 \%$ desse capital geraria um aumento (redução) médio de $0,24 \%$ do valor de produção.

Dentro das variáveis explicativas do modelo, se observa que a mão-de-obra é quem tem maior impacto sobre o valor de produção do setor agropecuário nortista. Pois a variação $1 \%$ na quantidade de mão-de-obra dos estabelecimentos poderia gerar um impacto médio de $0,60 \%$ na variável dependente, o que demostra a importância deste fator para a agropecuária da região. A variável investimento, em contraste, é que menos impacto tem sobre a variação relativa do valor de produção, 
pois o aumento de $1 \%$ no investimento geraria uma variação positiva de aproximadamente 0,09\% em valor médio de produção.

O coeficiente de $\mathrm{y}$ representaria o impacto que o termo de erro das regiões $j$ tem sobre o valor de produção agropecuário de uma determinada região $i$. Este fenômeno seria explicado por características intrínsecas da distribuição espacial e aglomeração de setor agrário, tal como afirma Almeida (2005). Ou seja, a dependência espacial da região Norte estaria associada a variáveis culturais, ambientais e climatológicas.

\section{Considerações Finais}

O setor agropecuário certamente é um elemento importante na geração de renda, emprego e desenvolvimento econômico dos países. Em virtude disso, este trabalho analisou o comportamento da agropecuária da região Norte através da análise explanatória de dados espaciais e a estimação de modelos econométricos espaciais.

Os resultados obtidos permitiram identificar que a região Norte possui baixa produtividade no setor agropecuário, principalmente nos municípios dos estados de Amazonas, Acre, Amapá e Roraima. Isto devido ao fato de que o valor de produção agropecuário dos estabelecimentos para o ano 2006 esteve concentrado no intervalo de $R \$ 1.188,21$ e $R \$ 553.302,17$

Constatou-se também que a região apresenta 4 tipos de clusters, sendo o tipo baixo-baixo (BB) o mais frequente. Os clusters com esta característica estão localizados nos estados de Amazonas, Acre e Amapá. A reduzida densidade rodoviária na região dificulta a acessibilidade e difusão de tecnologia entre os municípios.

Os testes específicos mostraram que existe dependência espacial no termo de erro no setor agropecuário da região Norte. Portanto, rejeita-se a hipótese de que o volume de produção de um determinado município não depende dos seus vizinhos. Todavia, esta dependência não se encontra nem na variável dependente nem na variável de interesse deste trabalho, senão no termo de erro, portanto, o melhor modelo para os dados da região Norte é o SMA.

Dessa forma, se obteve que na região de estudo não existe efeito de transbordamento de tecnologia. Isto confirma a hipótese que de a falta de proximidade, acessibilidade e semelhança sociocultural entre os estabelecimentos 
agropecuários da região não permitem o compartilhamento e difusão de tecnologia entre eles.

Com SMA, se obteve que as variáveis explicativas do modelo tiveram os sinais esperados, e que a quantidade de mão-de-obra dos estabelecimentos tem maior impacto sobre o valor de produção dos municípios da região norte quando comparados às outras variáveis. Em contraste, o impacto da variável investimento foi o menor. Ainda com os resultados da regressão pode-se afirmar que o valor de produção dos estabelecimentos nortista depende da média do termo de erro dos seus vizinhos, os quais podem estar relacionados com variáveis especificas de cada município, como a qualidade do solo, o clima etc.

Assim, uma das limitações deste trabalho foi a não inclusão de variáveis que permitam capturar as especificidades de cada estado da região Norte, tal como temperatura. Portanto, recomenda-se para trabalhos futuros tentar mensurar variáveis relativas a cultura, meio ambiente e clima e qualidade do solo de cada estado.

\section{REFERÊNCIAS}

ALMEIDA, E. Econometria Espacial Aplicada. Campinas: Alínea, 2012.

ALMEIDA, E. Função de Produção Agropecuária Espacial. In: Congresso Brasileiro de Economia e Sociedade Rural, Ribeirão Preto, 2005. Anais... Ribeirão Preto: Sociedade Brasileiro de Economia e Sociedade Rural (SOBER), 2005. CD-ROM.

ANSELIN, L. Local Indicators of Spatial Association-LISA. Geographical Analysis, v. 27, n.2, 1995.

ANSELIN, L. Spatial Dependence in Linear Regression Models with an Introduction to Spatial Econometrics. In: Ullah A. and Giles D.E. Handbook of Applied Economic

Statistics, Marcel Dekker, New York, p. 237-289, 1998.

ANSELIN, L. Spatial Econometrics. In: Badi H. Baltagi (Org.). A companion to Theoretical Econometrics, p. 310-330. 2003

BANCO MUNDIAL. Agricultura, Valor Agregado (\% del PIB). Disponível em: < https://datos.bancomundial.org/indicador/NV.AGR.TOTL.ZS?end=2016\&locations=BR\&start= 2000\&view=chart>. Acessado em: 28 de fevereiro de 2018.

BOTTI, E.A. O papel do agronegócio brasileiro no seu desenvolvimento econômico. Revista GEPROS. São Paulo, n 3, p. 149-158, 2014

CASTRO, N. Custo de Transporte e Produção Agropecuária no Brasil, 1970, 1996. Revista Agricultura em São Paulo. São Paulo, vol. 49, n. 2, pp. 87-109, 2002.

DIAS, C. A F.; OLIVEIRA, N. M. Estudo da Função de Produção Agropecuária do Estado de Goiás. In: Congresso Brasileiro de Economia e Sociedade Rural, Cuiabá, 2004, Anais... Cuiabá: Sociedade Brasileiro de Economia e Sociedade Rural (SOBER), 2004. CD-ROM. 
FREITAS, Eduardo de. "A Região Norte abriga a Amazônia"; Brasil Escola. Disponível em $<$ http://brasilescola.uol.com.br/brasil/a-regiao-norte-abriga-amazonia.htm>. Acesso em $09 \mathrm{de}$ maio de 2017.

GOLGHER, A.B. Introdução à Econometria Espacial. 1 ed. São Paulo: Editora Paco, 2015.

IBGE- INSTITUTO BRASILEIRO DE GEOGRAFIA E ESTATÍSTICA. Cidades@. Disponível em: < http://cidades.ibge.gov.br/xtras/home.php?lang=>. Acessado em 03 de janeiro de 2017.

IBGE- INSTITUTO BRASILEIRO DE GEOGRAFIA E ESTATÍSTICA. SIDRA, PRODUTO INTERNO BRUTO DOS MUNICIPIOS. Disponível em: <https://sidra.ibge.gov.br/tabela/5938 $>$. Acessado em 28 de fevereiro de 2018a.

IBGE- INSTITUTO BRASILEIRO DE GEOGRAFIA E ESTATÍSTICA. SIDRA, ESTIMAÇÃO DA POPULAÇÃO DOS ESTADOS. Disponível em: <https://sidra.ibge.gov.br/tabela/6579>. Acessado em 28 de fevereiro de 2018b.

IBGE- INSTITUTO BRASILEIRO DE GEOGRAFIA E ESTATÍSTICA. SIDRA, CENSO AGROPECUÁRIO 2006: SEGUNDA APURAÇÃO. Disponível em:

$<$ https://sidra.ibge.gov.br/tabela/765>. Acessado em 20 de agosto de 2018c.

IBGE- INSTITUTO BRASILEIRO DE GEOGRAFIA E ESTATÍSTICA. Contas Regionais do Brasil 2010-2014. Coordenação das Contas Nacionais. Rio de Janeiro, 2016

IBGE- INSTITUTO BRASILEIRO DE GEOGRAFIA E ESTATÍSTICA. Censo Agropecuário de 2017: resultados preliminares. IBEG: Rio de Janeiro, 2017

KAHNEMAN, D. Rápido e devagar: duas formas de pensar. 1 ed, Rio de Janeiro: objetiva, 2012

MANKIW, N.G. Princípios de Economia. 6 ed. Mexico, D.F: Cengage Learning, 2012.

MAZOYER, M.; ROUDART, L. História das Agriculturas no Mundo: do Neolítico à crise contemporânea. Marcel Mazoyer, Laurence Roudart; tradução de Cláudia F. Falluh, Balduino Ferreira. São Paulo, Editora UNESP; Brasília, DF: NEAD, 2010.

PEROBELLI, F. S.; ALMEIDA, E. S.; ALVIM, M. I. S.; FERREIRA, P. G. C. A. Análise Espacial da Produtividade do Setor Agrícola Brasileiro: 1991-2003. In: Congresso Brasileiro de Economia e Sociologia Rural, 43, Ribeirão Preto. Anais... Ribeirão Preto: Sociedade Brasileira de Economia e Sociologia Rural (SOBER), CD-ROM, 2005.

PINHEIRO, M.A; PARRÉ, J.L; LOPES, R.L. Um Estudo Exploratório sobre os Efeitos Espaciais na Agropecuária Paranaense In: Congresso Brasileiro de Economia e Sociologia Rural, 44, Fortaleza. Anais... Fortaleza: Sociedade Brasileira de Economia e Sociologia Rural (SOBER), CD-ROM, 2006.

ROCHA, C. B.; PARRÉ, J. L. Estudo da Distribuição Espacial do Setor Agropecuário do Rio Grande do Sul. Revista Análise Econômica. Porto Alegre, v. 27, n. 52, p. 139-160, set. 2009.

SANTOS, D. B. Diversificação e Especialização Produtiva na Geração de Inovação Tecnológica: Uma Aplicação para os Estados Brasileiros. 2014, p. 117. Dissertação (Mestrado em Desenvolvimento Econômico) - Departamento de Economia, Universidade Federal do Paraná, Curitiba, PR, mar. 2014. 
SILVA, M. V. S.; NONNENBERG, M.J. B. A Participação do Agronegócio no PIB Brasileiro: Controvérsias Conceituais e Propostas Metodológicas. In: Congresso Brasileiro de Economia e Sociedade Rural, Fortaleza, 2006, Anais... Fortaleza: Sociedade Brasileiro de Economia e Sociedade Rural (SOBER), 2006. CD-ROM.

SILVA, R.G; FERNANDES, E.A. Índice Relativo de Modernização Agrícola na Região Norte. Revista de Economia e Agronegócio. Viçosa, v. 3, n. 1, p. 29-50, 2005.

SILVA, R.R; BACHA, C.J.C. Acessibilidade e aglomerações na Região Norte do Brasil sob o enfoque da Nova Geografia Econômica. Revista Nova Economia. Belo Horizonte, v. 24, n.2, p. 169-190. Jan./abr. 2014.

VARIAN, H.R. Microeconomia: princípios básicos, uma abordagem moderna. 7 ed. Rio de Janeiro: Elsevier, 2006.

ZAMBERLAN, J.; FRONCHETI, A. Agricultura Ecológica: preservação do pequeno agricultor e o meio ambiente. 2 ed. Petrópolis: Vozes, 2001.

BARBOSA FILHO, F.H.; PESSÔA, S.A. Educação e Crescimento: O que a Evidência Empírica e Teórica Mostra? Revista EconomiaA. v. 11, n. 2, p. 265-303, 2010

GLAESER, E. L.; KALLAL, H. D.; SCHEINKMAN, J. A.; SHLEIFER, A. Growth in

Cities. Journal of political economy, v. 100, n. 6, p. 1126-1152, 1992.

FERREIRA, Z.R. Determinantes da Irrigação no Brasil: Uma Análise do Spillover Espacial. 2015. Dissertação (Mestrado em Economia Aplicada) - Departamento de Economia Rural, Universidade Federal de Viçosa, Viçosa, MG, 2015.

\section{NOTAS DE AUTOR}

\section{CONTRIBUIÇÃO DE AUTORIA}

Edi Flores Reyna - Concepção. Coleta de dados, Análise de dados, Elaboração do manuscrito, revisão e aprovação da versão final do trabalho

Rubicleis Gomes da Silva -Participação ativa da discussão dos resultados; Revisão e aprovação da versão final do trabalho.

Viviani Silva Lírio -Participação ativa da discussão dos resultados; Revisão e aprovação da versão final do trabalho.

\section{FINANCIAMENTO}

Apoio financeiro do Conselho Nacional de Desenvolvimento Científico e Tecnológico (CNPq) na forma de bolsa.

\section{CONSENTIMENTO DE USO DE IMAGEM \\ "Não se aplica"}

\section{APROVAÇÃO DE COMITÊ DE ÉTICA EM PESQUISA}

Não se aplica.

\section{CONFLITO DE INTERESSES}

Não se aplica.

\section{LICENÇA DE USO}

Este artigo está licenciado sob a Licença Creative Commons CC-BY. Com essa licença você pode compartilhar, adaptar, criar para qualquer fim, desde que atribua a autoria da obra. 